\title{
Multi-Objective Day-Ahead Optimal Scheduling of Isolated Microgrid Considering Flexibility
}

\author{
Liang Zhang ${ }^{1}$, Bo Pang ${ }^{1}$, Ruipeng $\mathrm{Yi}^{1}$, Pengyu Gai ${ }^{1}$, Chunqing Xin ${ }^{1}$, Longjie Yang ${ }^{2, *}$ and Huaqiang $\mathrm{Li}^{2}$ \\ ${ }^{1}$ State Grid Binzhou Power Supply Company, No.521 Yellow River Fourth Road, Binzhou, Shandong Province, China \\ ${ }^{2}$ Sichuan University, No.24 South Section 1, Yihuan Road, Chengdu, Sichuan Province, China
}

\begin{abstract}
In isolated microgrid, renewable energy sources including photovoltaic and wind power, have the nature of intermittence and variability. Making a reasonable day-ahead generation schedule could improve system ability to cope with uncertainty. Therefore, based on day-ahead generation schedule, flexibility insufficiency rate is proposed considering economy and flexibility. Aiming at the lowest flexibility insufficiency rate and optimal operating cost, a day-ahead generation schedule optimizing model of isolated microgrid is established. Under multi-objective particle swarm optimization, Pareto optimal solution set of the day-ahead generation schedule is found. Simulation results of an isolated microgrid show that, day-ahead generation schedule made with the proposed method can improve ability of power system to cope with uncertainty and reduce economic losses.
\end{abstract}

\section{Introduction}

Isolated microgrid is generally applied in remote areas or islands. Such micro-grids are not supported by large power grids, and are limited by their own capacity and uncertainties in the output of renewable energy, making it difficult to ensure real-time power balance [1]. Therefore, a reasonable day-ahead generation schedule can effectively improve the flexibility, reliability, and reduce costs of isolated micro-grid operations, utilization rate of renewable energy can be augmented. Literature [2] considers the operating cost of the micro-grid is most assured when the hybrid energy storage system balances the power deviation between real-time scheduling and day-ahead forecasting. In this connection, investment cost of the energy storage per unit is high, and the capacity change will affect the system operation, and the scheduling strategy is relatively complex.

In order to enhance the flexibility of isolated microgrid and its capacity to respond to uncertainties, this paper has established a flexibility insufficiency rate(FIR), while taking into consideration the running costs for the day-ahead generation schedule and the flexibility of system. Following that, the paper sets up a dispatch model for optimizing isolated microgrid. Aiming at minimizing the running costs, FIR has outlined a more proper day-ahead generation schedule, thus enabling a more flexible, reliable and economical system.

\section{Dispatch flexibility of microgrid}

How well the system of isolated micro-grids can adjust its process depends on flexible resources such as diesel

\footnotetext{
* Corresponding author: scuyanglongjie@foxmail.com
}

engine(DE), energy storage system(ESS) and demand side resources. Uncertainties faced by the operation are mainly related to wind power output, photovoltaic output and load fluctuations. In this context, the paper defines the dispatch flexibility of micro-grids as the capability of micro-grids to bring down the load fluctuation and handicap the process of discard winding, light, and load, by more properly mobilizing all kinds of flexible resources and reserving certain flexibility, during every dispatch period of day-ahead generation schedule, and amid fluctuations of wind power output, photovoltaic output and load output.

To guarantee the effectiveness of day-ahead generation schedule and make distributed power output and load consistent with the day-ahead generation schedule, this paper chooses DE as a flexible resource. The orientation of flexibility means that during every dispatch period, DE can make upward and downward adjustments. Such being said, the flexibility margin of DE during every period can be divided as upward and downward flexibility margin, and different margins are defined as this below:

$$
\left\{\begin{array}{l}
f_{m}^{\text {up }}(t)=\min \left(P_{m}^{\max }-P_{m}(t), \Delta t \cdot R_{m}^{\text {up }}, \Delta t \cdot R_{m}^{\text {up }}-\Delta P_{m}\right) \\
f_{m}^{\text {down }}(t)=\min \left(P_{m}(t)-P_{m}^{\text {min }}, \Delta t \cdot R_{m}^{\text {down }}, \Delta t \cdot R_{m}^{\text {down }}+\Delta P_{m}\right)
\end{array}\right.
$$

In this equation, $P_{m}^{\max }, P_{m}^{\min }$ and $P_{m}(t)$ are the maximum output power, the minimum output power, and the real-time output power of the time respectively for DE that ranks $m . \Delta t$ is defined as the scheduling interval; $R_{m}^{\text {up }}$ and $R_{m}^{\text {down }}$ are the up and down ramp power rates respectively for the DE that ranks $\mathrm{m} ; \Delta P_{m}$ is the power changed from period to period by the DE that ranks $m$. 
In each scheduling period, the DE should not only have a certain degree of flexibility, but also have the ability to return to the previous planned output during the next scheduled period when its output deviates from the previous schedule. Therefore, in each scheduling period, actual upward flexibility margin and downward flexibility margin are respectively $f^{\mathrm{ap}}(t)$ and $f^{\text {down }}(t)$.

$$
\left\{\begin{array}{l}
f^{\text {up }}(t)=\sum_{m=1}^{M} \min \left(f_{m}^{\text {up }}(t),\left(P_{m}(t+1)-P_{m}(t)+\Delta t \cdot R_{m}^{\text {up }}\right)\right) \\
f^{\text {down }}(t)=\sum_{m=1}^{M} \min \left(f_{m}^{\text {down }}(t),\left(P_{m}(t)-P_{m}(t+1)+\Delta t \cdot R_{m}^{\text {down }}\right)\right)
\end{array}\right.
$$

In the equation, $M$ is the total amount of $\mathrm{DE}$ in micro-grid system.

In order to measure the flexibility of micro-grid system, this paper has established an index for measurement considering to what extent flexibility is in shortage.

$$
\left\{\begin{array}{l}
F_{\mathrm{FR}}^{\mathrm{up}}=\sum_{t=1}^{T} \max \left(\frac{P^{\mathrm{down}}(t)-f^{\mathrm{up}}(t)}{P_{\mathrm{all}}^{\mathrm{down}}}, 0\right) \times 100 \% \\
F_{\mathrm{FR}}^{\text {down }}=\sum_{t=1}^{T} \max \left(\frac{P^{\mathrm{up}}(t)-f^{\text {down }}(t)}{P_{\mathrm{all}}^{\mathrm{up}}}, 0\right) \times 100 \%
\end{array}\right.
$$

In the equation, $F_{\mathrm{FIR}}^{\text {up }}, F_{\mathrm{FIR}}^{\text {down }}$ represent the upward flexibility and downward flexibility of mirco-grid system within a day respectively. $T$ is identified as the adjustment period. $P^{\text {down }}(t), P^{\text {up }}(t)$ are the maximum downward fluctuation power and the maximum upward fluctuation power within $t$ period. $P_{\text {all }}^{\text {down }}, P_{\text {all }}^{\text {up }}$ are the aggregate volume of downward and upward fluctuation power within a day.

The physical meaning of this indicator reflects the ability of the micro-grid system to stabilize the fluctuation of real-time wind power output, photovoltaic output and load output. The smaller the value is, the more adequate the system flexibility margin is, and the stronger the ability to suppress fluctuations. For example, when the sum of $F_{\mathrm{FIR}}^{\mathrm{up}}$ and $F_{\mathrm{FIR}}^{\mathrm{down}}$ is 0 , the system flexibility margin is sufficient, and the fluctuation of real-time scenery and load can be completely suppressed. When the sum is $100 \%$, the system will have no flexibility margin, if the scenery or load fluctuate, the system will be forced to abandon wind, light or load.

\section{Optimized day-ahead generation schedule for microgrid}

This paper takes the island-type micro-grid as the research object, including wind generators, photovoltaic arrays, DE, energy storage cells and loads.

\subsection{Micro-source model}

\subsubsection{DE model}

The relationship between fuel cost and output power during each DE period is

$$
C_{\mathrm{f} . \mathrm{DE}}(t)=\xi_{\mathrm{f} . \mathrm{DE}} \cdot P_{\mathrm{DE}}(t) \cdot \Delta t
$$

In the formula: $C_{\mathrm{f} . \mathrm{DE}}(t)$ is the fuel cost of $\mathrm{DE}$ within $t$ period; $P_{\mathrm{DE}}(t)$ is the output power of $\mathrm{DE}$ for the period; $\xi_{\text {f.DE }}$ is the cost of fuel consumption per $\mathrm{kW} \cdot \mathrm{h} \mathrm{DE}$.

\subsubsection{Energy Storage Model}

The state of charge (SOC) of the ESS is an important decision variable in the charging and discharging process of the ESS. The formula is listed below:

$$
S_{\mathrm{ESS}}(t+1)=(1-\delta) S_{\mathrm{ESS}}(t)-\frac{\Delta t \cdot \eta_{\mathrm{ESS}}(t) \cdot P_{\mathrm{ESS}}(t)}{E_{\mathrm{ESS}}}
$$

In this equation, $S_{\mathrm{ESS}}(t)$ is the charge state of ESS for the period; $\delta$ stands for the self-discharge efficiency of ESS; $\Delta t$ represents the scheduling interval; $P_{\mathrm{ESS}}(t)$ is the charge and discharge power of ESS, and it is regulated that the charge is negative; $E_{\mathrm{ESS}}$ is the maximum capacity of ESS; $\eta_{\mathrm{ESS}}(t)$ represents the charge and discharge efficiency of ESS for the period, and its value is

$$
\eta_{\mathrm{ESS}}(t)= \begin{cases}\eta_{\mathrm{c}}, & P_{\mathrm{ESS}}(t)<0 \\ 1 / \eta_{\mathrm{d}}, & P_{\mathrm{ESS}}(t)>0\end{cases}
$$

In this equation, $\eta_{\mathrm{c}}$ is set as charge efficiency of ESS; $1 / \eta_{\mathrm{d}}$ stands for discharge efficiency of ESS.

The output power of the wind turbine mainly depends on the wind speed. The specific model can be seen in literature [3]; the photovoltaic output power mainly depends on the light intensity, the illumination area and other factors, and the specific model is listed in literature [4].

\subsubsection{Load model}

This article classifies the load into four categories: Extremely Important, Important, Transitional, and General. The load can be removed from the low to the high according to the actual situation. The load to be removed for each scheduling period $P_{\mathrm{IL}}(t)$ is

$$
P_{\mathrm{IL}}(t)=\max \left\{\Delta P_{\mathrm{W}}^{\text {down }}(t)-f^{\mathrm{up}}(t), 0\right\}
$$

In the formula, $\Delta P_{\mathrm{W}}^{\text {down }}(t)$ is the downward fluctuation power of scenery based on the daily forecast for the period; $f^{\mathrm{ap}}(t)$ is the upward flexibility margin of the time.

At present, the prediction accuracy of load is very high, which can reach more than $95 \%$. In order to simplify the model, this paper believes that the actual load value is equal to the predicted value.

\subsection{Objective Function}

This paper adopts a daily optimization scheduling model that considers the forecasting error of the scenery, and aims to minimize the integrated operation $\operatorname{cost} F_{1}$ and the insufficiency of the flexibility rate for a day of running microgrid.

$$
\left\{\begin{array}{l}
F_{1}=F_{\mathrm{f}}+F_{\mathrm{OM}}+F_{\mathrm{E}}+F_{\mathrm{SL}}+F_{\text {cut }}+F_{\mathrm{CCOFE}} \\
F_{2}=F_{\mathrm{rRR}}^{\text {up }} \cdot \omega+F_{\mathrm{rRR}}^{\text {down }} \cdot(1-\omega)
\end{array}\right.
$$

Among them, 


$$
\begin{aligned}
& F_{\mathrm{f}}=\sum_{k=1}^{K} \sum_{t=1}^{T} C_{\mathrm{f} . k}(t) \\
& F_{\mathrm{OM}}=\sum_{k=1}^{K} \sum_{t=1}^{T}\left(C_{\mathrm{OM} . k} \cdot P_{k}(t) \cdot \Delta t\right) \\
& F_{\mathrm{E}}=\sum_{k=1}^{K} \sum_{j=1}^{J} \sum_{t=1}^{T}\left(C_{k . j} \cdot P_{k}(t) \cdot \Delta t\right) \\
& F_{\text {cut }}=\sum_{q=1}^{Q} \sum_{t=1}^{T}\left(C_{\mathrm{RES} . q}(t) \cdot P_{\text {cut } q}(t) \cdot \Delta t\right)+ \\
& \sum_{t=1}^{T} \sum_{s=1}^{S}\left(C_{\mathrm{L} . s}(t) \cdot P_{\text {cut.s }}(t) \cdot \Delta t\right) \\
& F_{\mathrm{CCOFE}}=F_{\mathrm{I}}+F_{\mathrm{RES}}+F_{\mathrm{L}}
\end{aligned}
$$

In the formula: $F_{\mathrm{f}}$ is the total fuel cost for all types of distributed power sources for one day; $K$ is the total number of distributed power types; $C_{\mathrm{f} . k}(t)$ stands for the fuel costs for the distributed $k$ type power source for the period $t ; F_{\mathrm{OM}}$ is the one-day operation and maintenance costs for all types of distributed power sources; $C_{\mathrm{OM} . k}$ is the operating cost for the distributed $k$ type power source per $\mathrm{kW} \cdot \mathrm{h} ; P_{k}(t)$ is the generation power of the distributed $k$ type power supply in the time; $F_{\mathrm{E}}$ is the cost of pollutant treatment generated during the day when the distributed power supply is running; $J$ is the total number of the types of the pollutants; $C_{k, j}$ is the cost parameter of the emission of type $j$ pollutants of the distributed type $k$ power supply; $F_{\text {cut }}$ is the cost of cutting the load and renewable energy, $Q$ is the total number of renewable energy types; $C_{\text {RES.q }}(t)$ is the cut-off cost per kilowatthour for the q type renewable energy for the period; $P_{\text {cut. } q}(t)$ is the resection power of the $q$ type renewable energy for the period; $S$ is the total number of segments divided by the removable load, among which the compensation cost parameter will increase along with the augment of divided segments; $C_{\mathrm{L} . s}(t)$ is the compensation cost parameter of load when the number $s$ section is removed for the period; $P_{\text {cut } . s}(t)$ is the load value of the number s section for the period; $F_{\mathrm{CCOFE}}$ is the compensation cost of forecast error(CCOFE), which constitutes the economic compensation of micro-grid system in reducing the forecast error. The economic compensation fee includes the cost $F_{\mathrm{I}}$ of mobilizing flexible resources to stabilize the fluctuation of scenery, the penalty $F_{\mathrm{RES}}$ for abandoned renewable energy, and the penalty $F_{\mathrm{L}}$ for load shedding. The specific calculation expressions for the three costs are:

$$
\begin{aligned}
& F_{\mathrm{I}}=\sum_{k=1}^{K} \sum_{t=1}^{T}\left(C_{\mathrm{I} . k}(t) \cdot \Delta P_{k}(t) \cdot \Delta t\right) \\
& F_{\mathrm{RES}}=\sum_{q=1}^{Q} \sum_{t=1}^{T}\left(C_{\mathrm{RES} . q}(t) \cdot \Delta P_{q}(t) \cdot \Delta t\right) \\
& F_{\mathrm{L}}=\sum_{s=1}^{S} \sum_{t=1}^{T}\left(C_{\mathrm{L} \cdot s}(t) \cdot \Delta P_{s}(t) \cdot \Delta t\right)
\end{aligned}
$$

In the formula, $C_{\mathrm{I} . k}(t)$ is the unit cost of the distributed $k$ type power supply in the time; $\Delta P_{k}(t)$ is mobilization power when the distributed $k$ type power supply reduces the forecast error for the period; $\Delta P_{q}(t)$ is the power of the abandoned wind and light after the forecasting error of the $q$ type renewable energy is stabilized in the period;
$\Delta P_{s}(t)$ is the value of cut power of the $s$ section after reducing the prediction error for the period.

$\omega$ is the weight parameter of the insufficiency rate for the micro-grid system within one day, and to convert the insufficiency rate of the upward flexibility of the microgrid system and the insufficiency rate of the downward flexibility of the micro-grid system into one-day flexibility insufficiency rate for the micro-grid system, the equation will be:

$$
\omega=\frac{P_{\text {all }}^{\text {down }}}{P_{\text {all }}^{\text {up }}+P_{\text {all }}^{\text {down }}}
$$

\subsection{The constraint condition}

\subsubsection{The constraint for power equilibrium}

$$
P_{\mathrm{DE}}(t)+P_{\mathrm{ESS}}(t)=P_{\mathrm{L}}(t)-P_{\mathrm{IL}}(t)-P_{\mathrm{WT}}(t)-P_{\mathrm{PV}}(t)
$$

In the equation, $P_{\mathrm{L}}(t)$ is the total load for the time, $P_{\mathrm{WT}}(t)$ is the wind power for the period, $P_{\mathrm{PV}}(t)$ is the photovoltaic power for the period.

\subsubsection{The constraint for distributed power supply}

$$
\left\{\begin{array}{l}
P_{\mathrm{DE} \cdot \min } \leq P_{\mathrm{DE}}(t) \leq P_{\mathrm{DE} \cdot \max } \\
P_{\mathrm{DE}}(t)-P_{\mathrm{DE}}(t-1) \leq R_{\mathrm{DE} \cdot \max }^{\mathrm{up}} \\
P_{\mathrm{DE}}(t-1)-P_{\mathrm{DE}}(t) \leq R_{\mathrm{DE} \cdot \max }^{\text {down }}
\end{array}\right.
$$

In the equation, $P_{\mathrm{DE} \text { min }}$ is the minimum power generation volume of $\mathrm{DE} ; P_{\mathrm{DE} \text { max }}$ is the rated power for $\mathrm{DE} ; R_{\mathrm{DE} \text {.max }}^{\text {up }}$ is the maximum upward climbing rate of $\mathrm{DE} ; R_{\mathrm{DE} \text {.max }}^{\text {down }}$ is the maximum downward climbing rate of $\mathrm{DE}$.

\subsubsection{Battery Charge and Discharge Constraints}

$$
\left\{\begin{array}{c}
S_{\mathrm{ESS} . \min } \leq S_{\mathrm{ESS}}(t) \leq S_{\mathrm{ESS} . \max } \\
\left|P_{\mathrm{ESS}}(t)\right| \cdot \Delta t^{\prime} \leq 0.2 E_{\mathrm{ESS}}
\end{array}\right.
$$

In the formula, $S_{\text {ESS.min }}, S_{\text {ESS.max }}$ are the upper and lower limits of the ESS state of charge; $\Delta t^{\prime}$ refers to $1 \mathrm{~h}$. To prolong life, the ESS's charge and discharge capacity per hour should be less than $20 \%$ of its maximum capacity.

\subsubsection{Interruptible load capacity constraints.}

$$
0 \leq P_{\mathrm{IL}}(t) \leq P_{\mathrm{IL} \cdot \max }(t)
$$

In the formula, $P_{\text {IL.max }}(t)$ stands for the maximum capacity that can be cut for removable load.

\subsection{Optimization Methods}

This paper aims at minimizing FIR and operating cost, and uses a multi-objective particle swarm optimization algorithm [5] to solve the scheduling model. Combined with the optimization goal of this paper, the SOC of each scheduling period of energy storage is used as the decision variable, and the Pareto optimal solution set of the target is solved by the algorithm. 


\section{Example Simulation}

\subsection{Example overview}

This article uses HOMER software simulation to obtain the basic values of scenery resources and loads, and uses this as an input for simulation analysis. Firstly, through forecasting data, this paper outlines the current scheduling plan according to the optimization goal, and then apply it to the real-time situation for verification. The prediction of wind speed and light intensity and the real-time curve are shown in Figure 1.

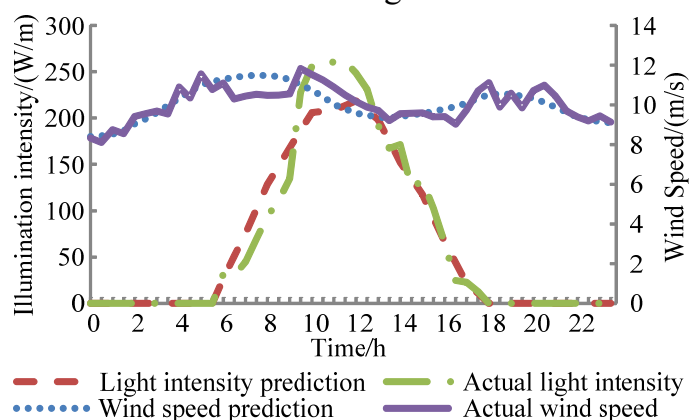

Fig. 1. Predicted curve and actual curve of wind speed and light intensity

The basic simulation parameters are as follows: The scheduling period is $30 \mathrm{~min}$; the cut-in wind speed $v_{\mathrm{i}}$ is 4 $\mathrm{m} / \mathrm{s}$; the rated wind speed $v_{\mathrm{r}}$ is $12 \mathrm{~m} / \mathrm{s}$; the cut-out wind speed $v_{\mathrm{o}}$ is $20 \mathrm{~m} / \mathrm{s}$; the rated power $P_{\mathrm{r}}$ of the fan is $90 \mathrm{~kW}$. The basic parameters of the PV array are: photovoltaic power generation efficiency $\eta_{\mathrm{PV}}$ is $12 \%$; light irradiation area $S_{\mathrm{PV}}$ is $1500 \mathrm{~m}^{2}$. The DE minimum power generation is $30 \mathrm{~kW}$; the DE rated power generation is $180 \mathrm{~kW}$; the DE maximum upward climbing power $R_{\mathrm{DE}}^{\text {up }}$ is $80 \mathrm{~kW} / \mathrm{h}$, and the DE maximum downward climbing rate $R_{\mathrm{DE}}^{\mathrm{down}}$ is $80 \mathrm{~kW} / \mathrm{h}$. The energy storage system has a rated capacity of $200 \mathrm{~kW} \cdot \mathrm{h}$. The state of charge changes from 0.2 to 0.9 and the maximum charge and discharge power is $40 \mathrm{~kW}$. The cost parameter of each power generation unit is shown in Table 1.

Table 1. Cost parameters of power generating unit

\begin{tabular}{|c|c|c|}
\hline Generation unit type & $\begin{array}{c}\text { Fuel cost / } \\
\left(¥ \cdot(\mathrm{kW} \cdot \mathrm{h})^{-1}\right)\end{array}$ & $\begin{array}{c}\text { Operation } \\
\text { management } \\
\text { coefficient / } \\
\left(¥ \cdot(\mathrm{kW} \cdot \mathrm{h})^{-1}\right)\end{array}$ \\
\hline Photovoltaic array & - & 0.0096 \\
\hline Wind driven generator & - & 0.0296 \\
\hline Energy storage battery & - & 0.0322 \\
\hline Diesel generator & 0.396 & 0.0880 \\
\hline
\end{tabular}

Table 2. Parameters of pollutant discharge and green gases

\begin{tabular}{|c|c|c|c|}
\hline \multicolumn{2}{|c|}{ pollutant type } & $\mathrm{CO} 2$ & $\mathrm{SO} 2$ \\
\hline \multicolumn{2}{|c|}{ handling expense $/\left(¥ \cdot \mathrm{kg}^{-1}\right)$} & 0.21 & 14.842 \\
\hline $\begin{array}{c}\text { pollutant } \\
\text { discharge } \\
\text { coefficient } \\
/\left(\mathrm{g} \cdot(\mathrm{kW} \cdot \mathrm{h})^{-1}\right)\end{array}$ & $\begin{array}{c}\text { photovoltaic power } \\
\text { generation }\end{array}$ & 0 & 0 \\
\cline { 2 - 4 } & wind power generation & 0 & 0 \\
\hline
\end{tabular}

Table 3. Cost of abandoning wind, abandoning light and cutting load

\begin{tabular}{|c|c|}
\hline $\begin{array}{c}\text { Abandon/ } \\
\text { Cut off the resource type }\end{array}$ & $\begin{array}{c}\text { Compensation costs / } \\
\left(¥ /(\mathrm{kW} \cdot \mathrm{h})^{-1}\right)\end{array}$ \\
\hline Wind & 0.60 \\
\hline Light & 0.85 \\
\hline Extremely important load & Unresectable \\
\hline Important load & 0.98 \\
\hline The transition load & 0.68 \\
\hline Non-essential load & 0.63 \\
\hline
\end{tabular}

The pollutants and greenhouse gas emission factors produced by DE operation are shown in Table 2; the costs of wind abandonment, light elimination and load shedding are shown in Table 3.

\subsection{Simulation and Result Analysis}

According to the typical day prediction curve, the maximum prediction error of wind and light is considered to be $20 \%$.After the operation, the curve of the FIR and the Pareto solution of the operating cost can be obtained as shown in Figure 2.

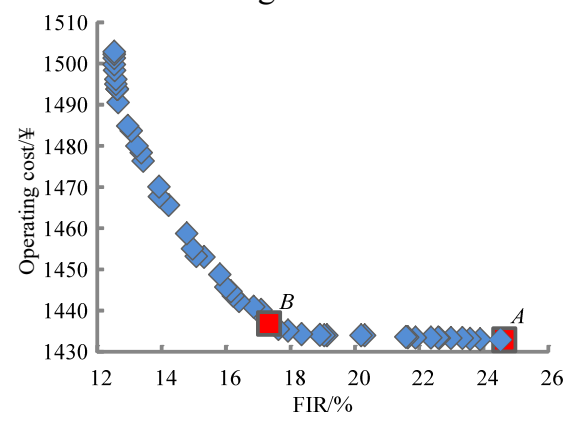

Fig. 2. Pareto solution for the Flexibility Insufficiency Rate and operating costs

This paper selects the daily scheduling plan A that considers only the optimal operating cost and the daily scheduling plan $\mathrm{B}$ that considers both the FIR and the operating costs to be optimal in Figure 2, and obtains the output of the DE and the energy storage plan, and then select the two kinds of day-ahead generation schedule A and $B$ which are calculated in real-time scenario. The results of DE real-time output are obtained, as shown in Figure 3 and Figure 4.

The real-time conditions of wind rejection, light rejection, and load shedding for Plan A and Plan B are shown in Figure 5. Table 4 shows the simulation results of the planned and real-time operating conditions.

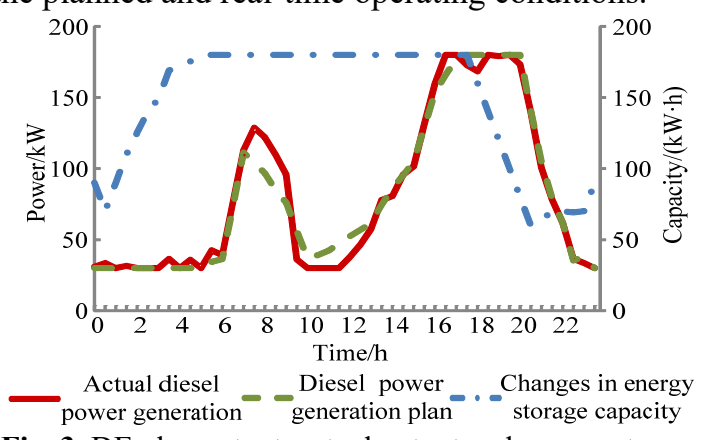

Fig. 3. DE plan output, actual output and energy storage capacity change of A scheme 


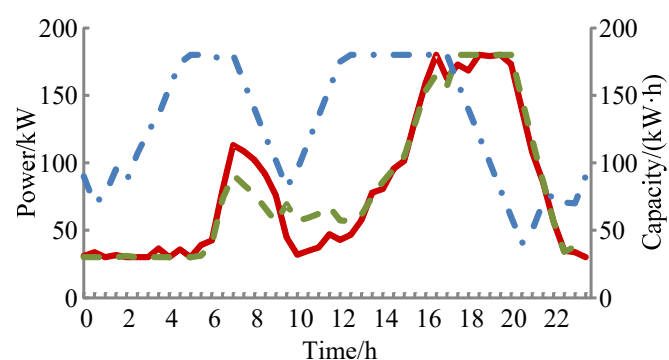

_ Actual diesel _ $\ldots$ Diesel power _. Changes in energy power generation generation plan storage capacity
Fig. 4. DE plan output, actual output and energy storage capacity change of $\mathrm{B}$ scheme

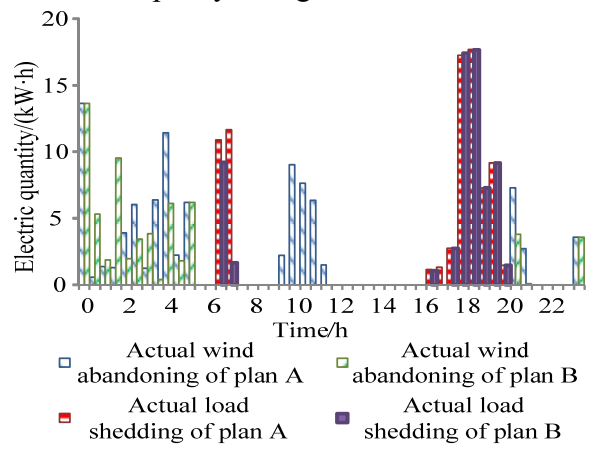

Fig. 5. The actual situation of abandoning wind, abandoning light and cutting load of $\mathrm{A}$ and $\mathrm{B}$ scheme

Table 4. Simulation data results of A and B scheme

\begin{tabular}{|c|c|c|}
\hline \multirow{2}{*}{ Quantities and units } & \multicolumn{2}{|c|}{ Plan } \\
\cline { 2 - 3 } & Plan A & Plan B \\
\hline Day-ahead FIR/\% & 24.64 & 17.34 \\
\hline Day-ahead operating cost/ $¥$ & 1432.90 & 1436.84 \\
\hline Real-time operating cost $¥$ \\
\hline $\begin{array}{c}\text { Day-ahead volume of wind } \\
\text { abandonment/(kW/h) }\end{array}$ & 1659.67 & 1628.47 \\
\hline $\begin{array}{c}\text { Day-ahead utilization rate of wind and } \\
\text { light/\% }\end{array}$ & 40.338 & 40.705 \\
\hline $\begin{array}{c}\text { Real-time volume of wind } \\
\text { abandonment/(kW·h) }\end{array}$ & 97.39 & 97.37 \\
\hline $\begin{array}{c}\text { Real-time utilization rate of wind and } \\
\text { light/\% }\end{array}$ & 93.91 & 96.03 \\
\hline $\begin{array}{c}\text { Day-ahead cutting load } /(\mathrm{kW} \cdot \mathrm{h}) \\
\text { Day-ahead rate of load cut } / \%\end{array}$ & 45.120 & 45.304 \\
\hline Real-time cutting load $/(\mathrm{kW} \cdot \mathrm{h})$ & 80.577 & 67.703 \\
\hline Real-time rate of load cut $/ \%$ & 2.32 & 1.95 \\
\hline
\end{tabular}

Fig. 2 - Fig. 5 and Table 4 are analyzed as follows:

1) As can be seen from Figure 2, FIR is the highest when considering only the optimal operating cost. As the FIR is reduced, the operating costs required are continuously increasing. In the end, increasing the operating cost can reduce the smaller amount of FIR. Therefore, there is a reasonable day-ahead generation schedule that can provide greater flexibility with smaller economic sacrifices, so that the system can better cope with the uncertainty in real-time operations and reduce the economic losses in real-time operations.

2) Compared with scheme $B$, scheme $A$ has low output, resulting in less downward flexibility of $\mathrm{DE}$ and higher FIR; in scheme B, to ensure that DE has a certain degree of flexibility margin, the stored energy is charged and discharged more frequently, making DE output as stable as possible within a certain range. Therefore, the FIR of the B scheme is $7.3 \%$ lower than that of the A scheme, but due to the increase of the DE output, the operating cost of the B scheme is slightly higher than that of the A scheme.

3) The total amount of typical abandonment wind planned for the A program is similar to that of the typical daily load, and the B plan is basically the same. Because the light abandon compensation fee is higher than the wind abandon compensation, both options are not abandoned; When the programs were run in real-time, the total amount of typical daily-shear loads of Plan B were reduced by $33.010 \mathrm{~kW} \cdot \mathrm{h}$ and $12.874 \mathrm{~kW} \cdot \mathrm{h}$ in wind abandonment and load abandonment, respectively, compared with Plan A. The real-time operating cost of Plan B was 31.20 yuan less than Plan A. In addition, the real-time wind and light utilization ratio of Plan A was $3.48 \%$ lower than the previous plan, and the load cut rate was increased by $1.02 \%$. The real-time scenery utilization rate of Plan B was $1.34 \%$ lower than the previous plan, and the load cut rate was increased by $0.64 \%$. Compared with the A program, it can better cope with fluctuations in the wind in the real-time situation, increase the utilization of renewable energy, and reduce the load. Therefore, it is more practical to consider operating expenses and FIR at the same time when formulating a day-ahead scheduling plan.

\section{Conclusion}

This paper aims at the dispatching operation of islandbased micro-grid and constructs the index of flexibility insufficiency. At the same time, it considers the optimal operating cost and the insufficiency of flexibility, and establishes a multi-objective optimization operation model of the system.

At the same time, this paper takes into account the operating costs and lack of flexibility, making the system better deal with the uncertainty in the real-time situation and reduce operating costs in real-time operation.

Therefore, in the formulation of the previous scheduling plan, it considers the lack of flexibility, the utilization rate of renewable energy, system economics, and the ability to cope with the uncertainty of scenery, which can be further increased and is of great significance to the actual operation of the system.

\section{References}

1. Yang M., Han X., Modern Electric Power, 32(4), 12-18 (2015).

2. Liu F., Yang X., Shi S., et al. Power System Technology, 38(11), 3079-3087 (2014).

3. Zhou W., Sun H., Gu H., et al. Proceedings of the CSEE, 32(1), 47-55 (2012).

4. Wang M Q, Gooi H B. IEEE Transactions on Power Systems, 26(3), 1164-1174 (2011).

5. Dou X., Ji S., Li Y., et al. Modern Electric Power, 32(5), 42-47 (2015). 